In S. Cotin and D.N. Metaxas, eds., "Proceedings of Medical Simulation:

International Symposium - ISMS 2004, Cambridge, MA, June 17-18, 2004,

Lecture Notes in Computer Science vol. 3078, Springer-Verlag, pp. 67-76.

\title{
A Nonlinear Finite Element Model of Soft Tissue Indentation
}

\author{
Yi Liu ${ }^{1}$, Amy E. Kerdok ${ }^{1,2}$, Robert D. Howe ${ }^{1,2}$ \\ ${ }^{1}$ Harvard University Division of Engineering and Applied Sciences \\ ${ }^{2}$ Harvard/MIT Division of Health Sciences and Technology \\ kerdok@fas.harvard.edu
}

\begin{abstract}
Mathematically describing the mechanical behavior of soft tissues under large deformations is of paramount interest to the medical simulation community. Most of the data available in the literature apply small strains $(<10 \%)$ to the tissue of interest to assume a linearly elastic behavior. This paper applies a nonlinear hyperelastic 8-chain network constitutive law to model soft tissues undergoing large indentations. The model requires 2 material parameters (initial modulus, locking stretch) to reflect the underlying physics of deformation over a wide range of stretches. A finite element model of soft tissue indentation was developed and validated employing this constitutive law. Ranges of the initial shear modulus and locking stretches were explored based on values found for breast tissue [17, 25]. Results of the model are shown with a lookup table containing third order polynomial coefficient fits. This work serves as an initial method to determine the unique material parameters of breast tissue from indentation experiments.
\end{abstract}

\section{Introduction}

Accurate mathematical descriptions of the mechanical behavior of soft tissues remain the limiting factor in the advancement of realistic medical simulations and noninvasive diagnostic tools. This is due to the complex nonlinear material properties of soft tissues when they undergo large mechanical deformations during minimally invasive procedures and diagnostic palpations.

A phenomenological approach is implemented to realize the material parameters of soft tissues. Inverse finite element modeling (FEM) is used to fit mathematical expressions in the form of a constitutive law to experimental data. Soft tissues are most often tested in an ex-vivo state with specimens of finite thickness under controlled loading and boundary conditions [8, 15, 18]. Selecting the appropriate constitutive law allows FEM to then be used to predict the tissue's response to modes of deformation not capable of being experimentally measured. We are interested in modeling the indentation of soft tissues by a rigid flat-ended cylindrical punch.

This paper provides a method for determining an initial estimate of the material parameters of soft tissue using the Arruda-Boyce constitutive model. A range of the two physically based material parameters of this model were explored based on data found in the literature on normal and pathologic breast tissue [17, 21, 22, 25]. The resulting force-nominal strain plots were fitted to $3^{\text {rd }}$ order polynomials whose 
2 Yi Liu1, Amy E. Kerdok1, 2, Robert D. Howe1, 2

coefficients and the resulting material parameters are presented. Others have attempted to model breast tissue under uniaxial compression and assumed linear elasticity [3, 20]. Han assumed quasilinear viscoelasticity with an exponential elastic response and modeled the breast under plain strain conditions because he used a rectangular shaped probe on thick specimens [10].

Results indicated here should serve as a means for identifying an estimate of the physiologically based material parameters of the ArrudaBoyce model.

\section{Background}

There exists a well-defined analytic solution for indentation by a rigid flat punch assuming infinitesimal strains, frictionless contact, and a semi-infinite incompressible elastic half-space [14, 24]. To account for the finite thickness in indentation experiments on cartilage, Hayes expanded the analytical solution to include a term that is dependant on the sample thickness:

$$
P=\frac{2 a E w}{\left(1-v^{2}\right)^{2}} \kappa\left(\frac{a}{h}, \frac{w}{h}\right)
$$

where $P$ is the applied force, $E$ is the elastic modulus, $a$ is the radius of the indenter, $w$ is the depth of indentation, and $\kappa$ is a dimensionless term to account for sample thickness (Fig. 1) [11]. Zheng created a finite element model using equation (1) to explore the effects of nonlinear geometry, namely large deformations up to $15 \%$ nominal strain, compressibility, and friction on the indentation of cartilage attached to a semi-infinite rigid half space [28]. Our goal is to further this approach by introducing both larger strains $(\sim 50 \%)$, and material nonlinearities into a FEM of soft tissue under indentation.

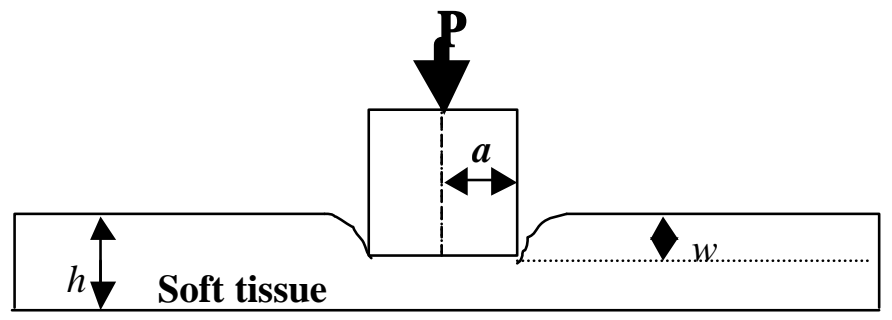

Fig. 1. Conceptual diagram of the soft tissue indentation model.

It is well understood that soft tissues are viscoelastic, anisotropic, inhomogeneous, and have nonlinear force displacement characteristics [9]. To simplify the mathematical analysis, many researchers assume an initial isotropy, local homogeneity, and study tissue deformations in the linear regime of $<10 \%$ nominal strain $[17,19,25]$. However, typical surgical manipulations are often much larger than $10 \%$ nominal strain. It has been shown that at larger strains an elastic contrast 
exists between tissues of different pathologic states [17, 25, 27]. Therefore more accurate representations of soft tissue behavior are needed.

Holzapfel suggests that only biological materials and solid polymers (rubber-like materials) undergo finite strains elative to an equilibrium state [12]. Therefore it should not be surprising that the hyperelastic constitutive models developed for elastomers have frequently been used to study soft tissues $[5,8,9,13,15,16,18,23]$. Hyperelastic materials are considered initially isotropic and exhibit a nonlinear instantaneous response up to large strains. There are two ways in which the strain energy functions for hyperelastic materials are derived: one based on continuum mechanics and the other based on statis tical mechanics.

We have created a FEM with a hyperelastic constitutive model based on statistical mechanics. We describe that model and the predictions it makes for large strain indentations of pathologic breast tissues.

\section{Materials and Methods}

\subsection{Creating and Validating the Finite Element Model}

Using commercial finite-element software (ABAQUS 6.3-1, HKS, Rhode Island), the present investigation created an axisymmetric rigid indenter model to analyze the indentation of soft tissue (Fig. 2). The model was validated against the analytical solution presented in equation 1 (with both $\kappa=1.0$ and $\kappa>1.0$ ) and compared to Zheng et al's [28] finite element model under infinitesimal strains before adding a nonlinear constitutive law.

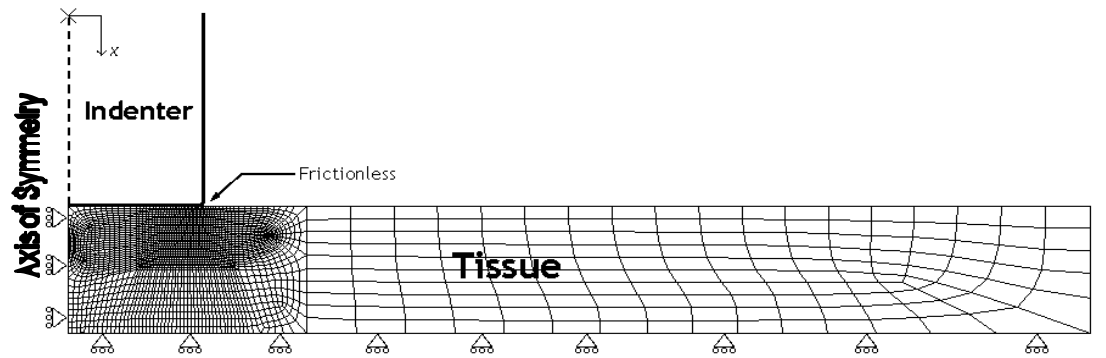

Fig. 2. Axisy mmetric FEM of rigid body indenter and soft tissue mesh with frictionless contact and sliding boundary conditions on the base.

The indenter was modeled as an analytical rigid body with a flat-ended cylindrical shape $12 \mathrm{~mm}$ in diameter. Initially the tissue (cartilage) was modeled as a deformable meshed layer and was assumed to be linearly elastic, isotropic, and incompressible with Young's modulus E $=100 \mathrm{kPa}$ and Poisson's ratio $\mathrm{v}=0.4999^{1}$ [28]. Its mesh consisted of 4 noded hybrid quadrilateral axisymmetric elements $(\mathrm{CAX} 4 \mathrm{H})$, finely biased in the immediate regions underneath the indenter as shown in Figure. 2. The

\footnotetext{
${ }^{1}$ Due to ABAQUS' limitations when $\mathrm{v}=0.5$, an incompressibility of $\mathrm{v}=0.4999$ was used.
} 
contact between the indenter and the tissue was modeled using a "contact pair" where the indenter was specified as "master" and the tissue as "slave." The contact property was defined as frictionless so that the tissue could freely slip beneath the indenter. Known displacements were then applied to the reference node of the indenter that was initially oriented on the surface of the tissue. The reaction forces generated by the FE simulation were recorded and plotted against strain.

To validate the model against the case of the true analytical solution of a rigid flat punch (equation 1 where $\kappa=1.0$ ), the boundary condition of the bottom surface is free. The tissue was unconstrained in the lateral direction and an aspect ratio (indenter radius to sample thickness) of 0.1 was used to approximate the seminfinite elastic half space. Results from the FEM und er $0.1 \%$ nominal strain are within $3.3 \%$ of the analytical form of the solution.

The FEM was modified to validate against Hayes' analytical model ( $\kappa>1.0$ in equation 1). Fixed boundary conditions simulated the attachment of cartilage to a rigid bony layer [11]. Two aspect ratios were tested $(0.2$ and 1.0$)$ to a strain of $0.1 \%$. Less than $2 \%$ error occurs when the FEM accounts for finite tissue thickness and is compared to both Hayes' analytical solution and Zhang's FEM results at nominal strains of $0.1 \%$ (Table 1 ).

Table 1. A comparison of the kappa value between our model (Liu) and the analytical solution of Hayes and the FEM of Zheng for 2 different aspect ratios.

\begin{tabular}{|c|c|c|c|}
\hline Aspect ratio & Model & $\kappa$ & \% Error \\
\hline \multirow{3}{*}{$\mathbf{a} / \mathbf{h}=\mathbf{0 . 2}$} & Liu & 1.260 & - \\
\cline { 2 - 4 } & Zhang & 1.244 & 1.25 \\
\cline { 2 - 4 } & Hayes & 1.281 & -1.67 \\
\hline \multirow{3}{*}{$\mathbf{a} / \mathbf{h}=\mathbf{1 . 0}$} & Liu & 3.564 & - \\
\cline { 2 - 4 } & Zhang & 3.590 & -0.72 \\
\cline { 2 - 4 } & Hayes & 3.609 & -1.24 \\
\hline
\end{tabular}

After the model was validated assuming linear elasticity under infinitesimal strains, the model was changed to simulate soft tissue indentation tests containing both geometric and material property nonlinearities. Experiments on breast tissue indentation found in the literature allow for frictionless contact between both the indenter and the specimen, and between the specimen and the testing surface $[17,25]$. Thus, the boundary condition on the bottom surface of the tissue in the model was unconstrained in the lateral direction. To compare to the experimental breast tissue dat a, the indenter size was changed to $4 \mathrm{~mm}$ in diameter and aspect ratios of $0.5,1.0$, and 1.5 were created $[17,25]$. The indenter fillet was increased to $2 \times 10^{-4} \mathrm{~mm}$ to allow the tissue to be compressed to $50 \%$ nominal strain The mesh bias was refined until a model of each aspect ratio could reach the set strain of $50 \%$. Strain rate tests performed on breast tissue suggest that viscous effects can be neglected [17, 25]. Hyperelastic nonlinear material parameters were added to the material property definitions of the tissue in the indentation model. Specifically, the Arruda-Boyce constitutive law was selected. 


\subsection{The Arruda-Boyce Constitutive Model}

\subsubsection{Motivation}

The continuum mechanics approach for developing hyperelastic strain energy functions are empirical need more than one experiment to realize their many material parameters, and have a limited strain region over which their results are applicable. Although higher order models fit the data well, they are complex, computationally expensive, and unstable at high stretches $[1,4]$. Despite these difficulties they are still widely used to describe the behavior of soft tissues [5, 8, 9, 13, 15, 16, 18, 23].

The statistical mechanics treatment of rubber elasticity (Langevin chain statistics) model the material chain segment between chemical cross-links as a rigid link with set length [4]. The stress-strain behavior is governed by changes in configurational entropy [2]. The end result reflects the underlying physics of macroscopic deformation from microscopic components. In particular the Arruda-Boyce model is an 8-chain network model where only two material parameters (the rubbery initial modulus and the limiting chain extensibility) are needed to describe the behavior of a material over a wide range of stretches given limited test data. This model lends itself ideally to that of soft tissues because the polymer chains mimic their main constituents: collagen and elastin fibers .

\subsubsection{Development}

A convenient form of the Arruda-Boyce strain energy function, $U$, is found by taking a series expansion of the inverse Langevin function to the $5^{\text {th }}$ order:

$$
U=\mu \sum_{i=1}^{5} \frac{C_{i}}{\lambda_{m}^{2 i-2}}\left(\overline{I_{1}^{i}}-3^{i}\right)+\frac{1}{D}\left(\frac{J_{e l}^{2}-1}{2}-\ln J_{e l}\right)
$$

where

$$
\begin{gathered}
C_{1}=\frac{1}{2}, C_{2}=\frac{1}{20}, C_{3}=\frac{11}{1050}, C_{4}=\frac{19}{7000}, C_{5}=\frac{519}{673750} \\
\mu=n \mathrm{~K} \theta \\
\lambda_{m}=\sqrt{N} \\
\bar{I}_{1}=\operatorname{tr}(\lambda)=\lambda_{1}^{2}+\lambda_{2}^{2}+\lambda_{3}^{2} .
\end{gathered}
$$

Here $\mu$ is the initial rubbery shear modulus, $n$ is the chain density, $\theta$ is the temperature, $K$ is Boltzmann's constant, $\lambda_{m}$ is the limiting chain extensibility (locking stretch), $N$ is the number of rigid links, $\mathrm{I}_{\mathrm{l}}$ is the first deviatoric strain invariant, and $\lambda_{\mathrm{i}}$ are the principle stretches. $\mathrm{D}$ is a temperature dependant material parameter related to the bulk modulus, and $\mathrm{J}_{\mathrm{el}}$ is the elastic volume ratio. For incompressible materials, $\mathrm{J}_{\mathrm{el}}$ $=1$ and the second term in equation 2 drops out.

Due to symmetry each chain's stretch is shared equally amongst all of the chains and an initially isotropic configuration can be assumed. We can therefore relate the microscopic chain length to the macroscopic principal stretches via: 


$$
\lambda_{\text {chain }}=\frac{1}{\sqrt{3}}\left(\sqrt{\lambda_{1}^{2}+\lambda_{2}^{2}+\lambda_{3}^{2}}\right)=\frac{l}{l_{0}}
$$

where $\lambda_{\text {ehain }}$ is the chain stretch, $l$ is the current chain length, and $l_{0}$ is the initial chain length. The locking stretch, $\lambda_{m}$, is the value of the chain stretch when $\dot{t}$ reaches its fully extended state, and can be determined from a simple tension or compression experiment assuming incompressibility $\left(\lambda_{1} \lambda_{2} \lambda_{3}=1\right)$. Modeling a uniaxial compression state and noting from the literature that breast tissue drastically increases its stress response at strains on the order of $30 \%$ nominal strain for normal tissue and $10 \%$ nominal strain for cancerous tissue, locking stretches were calculated to be 1.05 and 1.01 respectively.

Literature on breast tissue material property measurements of varying pathology suggest that initial elastic moduli for cancerous tissue is between 3 and 7 times that of normal tissue [17, 21, 22, 25]. For an incompressible material Possion's ratio is 0.5 and the elastic modulus is equal to 3 times the shear modulus. Given initial elastic moduli reported in the literature of $33 \mathrm{kPa}$ and $100-186 \mathrm{kPa}$ for normal and infiltrating ductal carcinoma respectively at 5\% nominal strain, we explored initial shear moduli between $1 \mathrm{kPa}$ and $150 \mathrm{kPa}$.

\subsection{Applying the nonlinear constitutive law to the FEM}

Using the proposed FE model, we chose to model eight different combinations of the two material parameters of the Arruda-Boyce constitutive law over three different aspect ratios $(\mathrm{a} / \mathrm{h}=0.5,1.0,1.5): \mu=1,5,10,20 \mathrm{kPa}$ with $\lambda_{\mathrm{m}}=1.05$, and $\mu=30,60$, $100,150 \mathrm{kPa}$ with $\lambda_{\mathrm{m}}=1.01$. The models were deformed to $50 \%$ nominal strain and the displacement and reaction force in the axis of deformation were recorded.

The Arruda-Boyce model is typically used for very large strains (i.e. tensile strains $>200 \%$ ). We are only interested in compressive strains on the order of $50 \%$. We can assume the effects from the higher order polynomial terms are therefore negligible. Third order polynomials were fit to the force-nominal strain curves generated from our FEM analysis. The coefficients of these polynomials can be compared to experimental data to estimate the material parameters of the substrate under study.

\section{Results}

The force-nominal strain responses of the FEM with different values of the initial shear modulus and locking stretch material parameters of the Arruda-Boyce constitutive model are shown below in Figure 4. Eight values of the initial modulus ranging from $1 \mathrm{kPa}$ to $150 \mathrm{kPa}$ were modeled with two values of the locking stretch (1.01 and 1.05) based on breast tissue data found in the literature as previously stated. Three aspect ratios were modeled to account for different sample thickness and indenter geometry. Typical computation times for $50 \%$ strain on a Pentium III computer were on the order of 140 seconds. The coefficients of third order polynomials fit to the model's response and their $\mathrm{R}^{2}$ values are shown in Table 2 . 
Table 2. The material parameters of the Arruda-Boyce model and their resulting third order polynomial fit coefficients for the force-nominal strain responses of soft tissue indentation.

$\mathrm{a} / \mathrm{h}=0.5$
\begin{tabular}{|c|c|c|c|c|c|}
\hline $\mathbf{R}^{\wedge} \mathbf{2}$ & A & B & C & lambda & mu (kPa) \\
\hline 0.9997 & 1.48 & 0.53 & 0.31 & 1.05 & 1 \\
\hline 0.9997 & 7.46 & 2.72 & 1.56 & 1.05 & 5 \\
\hline 0.9997 & 14.80 & 5.28 & 3.11 & 1.05 & 10 \\
\hline 0.9997 & 29.88 & 10.83 & 6.25 & 1.05 & 20 \\
\hline 0.9997 & 57.43 & 20.83 & 10.90 & 1.01 & 30 \\
\hline 0.9997 & 113.81 & 41.21 & 21.69 & 1.01 & 60 \\
\hline 0.9997 & 187.01 & 67.21 & 35.87 & 1.01 & 100 \\
\hline 0.9996 & 275.91 & 98.22 & 53.38 & 1.01 & 150 \\
\hline
\end{tabular}

$\mathrm{a} / \mathrm{h}=1.0$
\begin{tabular}{|c|c|c|c|c|c|}
\hline $\mathbf{R}^{\wedge} \mathbf{2}$ & $\mathbf{A}$ & $\mathbf{B}$ & $\mathbf{C}$ & lambda & $\mathbf{m u}(\mathbf{k P a})$ \\
\hline 0.9995 & 1.20 & 0.43 & 0.23 & 1.05 & 1 \\
\hline 0.9995 & 5.92 & 2.10 & 1.16 & 1.05 & 5 \\
\hline 0.9995 & 11.73 & 4.16 & 2.30 & 1.05 & 10 \\
\hline 0.9995 & 23.16 & 8.17 & 4.58 & 1.05 & 20 \\
\hline 0.9995 & 43.93 & 15.43 & 7.93 & 1.01 & 30 \\
\hline 0.9995 & 86.04 & 29.99 & 15.71 & 1.01 & 60 \\
\hline 0.9995 & 140.78 & 48.72 & 25.93 & 1.01 & 100 \\
\hline 0.9995 & 207.65 & 71.35 & 38.55 & 1.01 & 150 \\
\hline
\end{tabular}

$\mathrm{a} / \mathrm{h}=1.5$
\begin{tabular}{|c|c|c|c|c|c|}
\hline $\mathbf{R}^{\wedge} \mathbf{2}$ & A & B & C & lambda & $\mathbf{m u ~}(\mathbf{k P a})$ \\
\hline 0.9995 & 1.19 & 0.44 & 0.22 & 1.05 & 1 \\
\hline 0.9995 & 5.85 & 2.17 & 1.07 & 1.05 & 5 \\
\hline 0.9995 & 11.56 & 4.27 & 2.13 & 1.05 & 10 \\
\hline 0.9995 & 22.75 & 8.38 & 4.22 & 1.05 & 20 \\
\hline 0.9994 & 42.98 & 15.75 & 7.31 & 1.01 & 30 \\
\hline 0.9994 & 83.87 & 30.52 & 14.44 & 1.01 & 60 \\
\hline 0.9994 & 136.84 & 49.49 & 23.79 & 1.01 & 100 \\
\hline 0.9994 & 201.47 & 72.42 & 35.31 & 1.01 & 150 \\
\hline
\end{tabular}

\section{Conclusions and Future Work}

For realistic medical simulations to become a practical reality the acquisition of biomechanical information and efficient computation must be achieved. The latter is left to the many researchers working on deformable meshing techniques $[6,7,26]$. It was the intent of this paper to focus on uniquely characterizing the complex nonlinear behavior of soft tissues with a simple mathematical model given limited experimental data. We implemented such a model in finite element simulations to predict the behavior of soft tissues undergoing large indentation deformations across various testing geometries. The model was validated in the linear regime against analytical solutions and another FEM. The force-nominal strain results of the model can be used to estimate the material parameters of the soft tissue of interest.

An axisymmetric finite element model with frictionless contact and boundary conditions was created employing the hyperelastic Arruda-Boyce constitutive model. Unlike similar constitutive laws formulated from continuum mechanics, this statisical mechanics based model was chosen because its two material parameters have a physical interpretation that can be directly related to the constituent make-up of soft tissues (collagen and elastin fibers). 

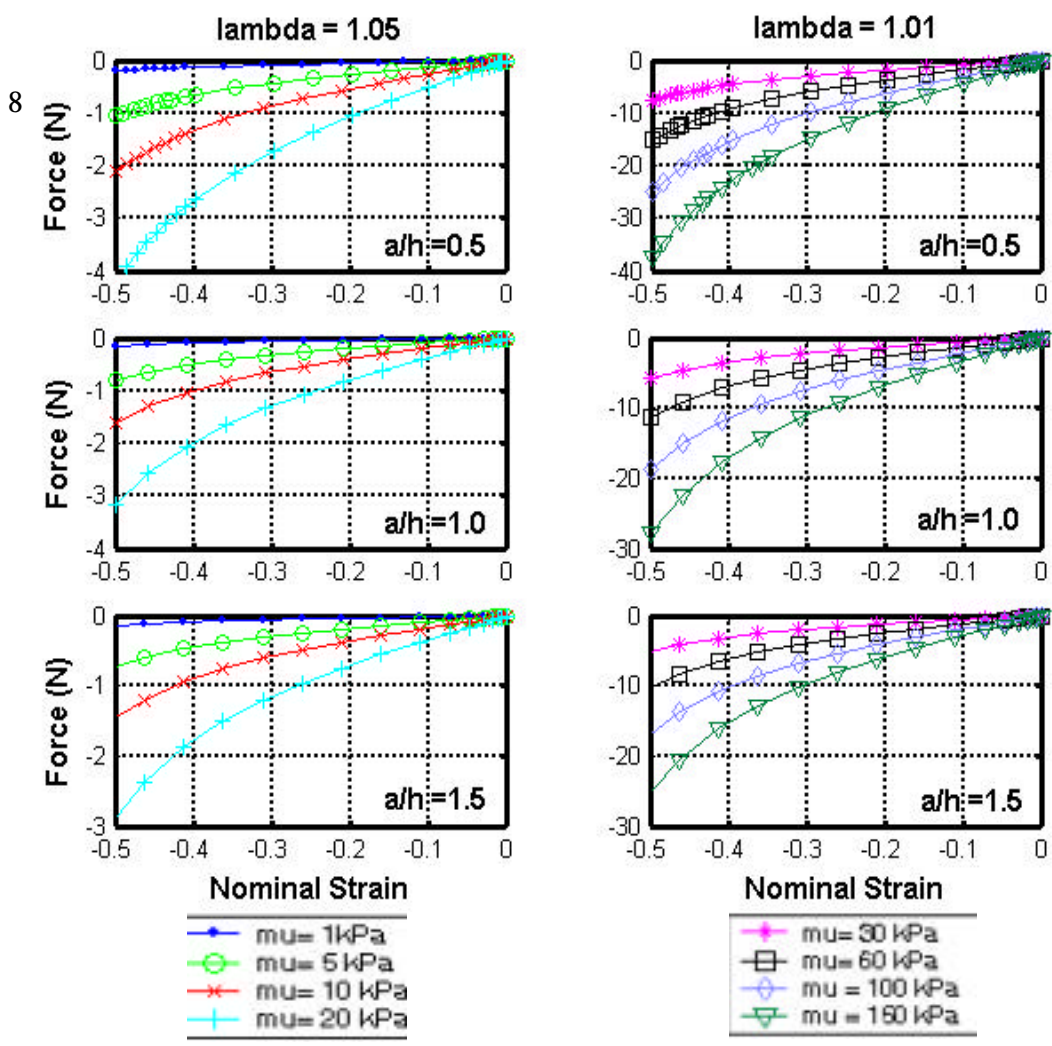

Fig. 4. Force versus nominal strain results for the FEM with $\mathrm{a} / \mathrm{h}=0.5$ (top), $\mathrm{a} / \mathrm{h}=1.0$ (middle), and $\mathrm{a} / \mathrm{h}=1.5$ (bottom) across various initial shear moduli and locking stretches $\left(\lambda_{\mathrm{m}}=1.05\right.$ (left) and $\left(\lambda_{\mathrm{m}}=1.01\right.$ (right)) up to $50 \%$ nominal strain.

Most of the soft tissue data published in the literature only apply nominal strains of up to $10 \%$. At these low strains, the Arruda-Boyce model reduces to the linear elastic Neo-Hookean form and fits the data well. At strains where the usefulness of these elastic models is of limited value the Arruda-Boyce model continues to predict the nonlinear behavior of the soft tissues.

Wellman has collected some indentation data on pathologic breast samples at larger strains $(>35 \%$ nominal strain). A future study will analyze this data and compare it to nonlinear FEM simulations to determine the unique material properties of the tissue. The lookup tables presented in this paper will be used to obtain approximate initial values for the material parameters. An iterative process will then ensue where the models' results will be compared to the large strain data via the employment a nonlin ear search scheme minimizing the sum of squares error. With an educated estimate of the initial value of the material parameters, convergence of a unique set of material parameters can be quickly obtained to within the standard error of the data collected.

A preliminary set of both normal glandular and cancerous data are plotted together with the corresponding FEM results in Figure 5. Fitting a third order polynomial to the data in Figure 5 suggests that an estimate for the normal glandular tissue material parameters are on the order of $\lambda_{\mathrm{m}}=1.05$ and $\mu=1 \mathrm{kPa}(\mathrm{a} / \mathrm{h}=1.0)$ and for infiltrating ductile carcinoma $\lambda_{\mathrm{m}}=1.01$ and $\mu=30 \mathrm{kPa}(\mathrm{a} / \mathrm{h}=1.5)$. 

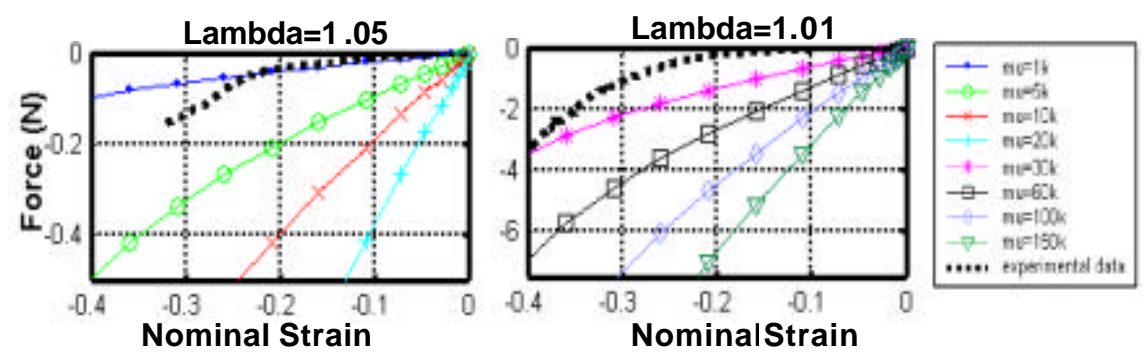

Fig. 5. Preliminary large strain indentation data plotted for normal breast tissue with $\mathrm{a} / \mathrm{h}=1.0$ (left) and infiltrating ductile carcinoma (right) against FEM results with $\mathrm{a} / \mathrm{h}=1.5$.

It is clear from this preliminary work that the model needs further development. The tissue appears to have a lower locking stretch than the Arruda-Boyce model predicts. This is most likely because the Arruda-Boyce model assumes an initial stress-free reference state, where as in real tissue this does not exist due to hydration and tension in the fibers. Accounting for this non-zero initial stress state should bring the model into closer agreement with the data and is currently being developed.

\section{References}

1. Anand, L.: A Constitutive Model for Compressible Elastomeric Solids. Computational Mechanics, 18. (1996) 339-355

2. Arruda, E.M.Boyce, M.C.: A Three-Dimensional Constitutive Model for The Large Stretch Behavior of Rubber Elastic Materials. J. Mech. Phys. Solids, 41. (1993) 389-412

3. Azar, F.S., Metaxas, D.N.Schnall, M.D.: A Deformable Finite Element Model of the Breast for Predicting Mechanical Deformations under External Perturbations. Journal of Academic Radiology, 8. (2001) 965-975

4. Boyce, M.C.Arruda, E.M.: Constitutive Models of Rubber Elasticity: A Review. Rubber Chemistry and Technology, 73. (2000) 504-523

5. Carter, F.J., Frank, T.G., Davies, P.J., McLean, D.Cuschieri, A.: Measurements and Modeling of the Compliance of Human and Porcine Organs. Medical Image Analysis, 5. (2001) 231-236

6. Cotin, S., Delingette, H.Ayache, N.: Real-Time Elastic Deformations of Soft Tissues for Surgery Simulation. IEEE Transactions on Visualization and Computer Graphics, 5. (1999) $62-73$

7. Delingette, H.: Towards Realistic Soft Tissue Modeling in Medical Simulation. In: IEEE: Special Issue in on Virtual and Augmented Reality in Medicine, 86. 1998) 12

8. Farshad, M., Barbezat, M., Flueler, P., Schmidlin, F., Graber, P.Niederer, P.: Material Characterization of the Pig Kidney in Relation with the Biomechanical Analysis of Renal Trauma. Journal of Biomechanics, 32. (1999) 417-425

9. Fung, Y.C.: Biomechanics: Mechanical Properties of Living Tissues. second. SpringerVerlag, New York (1993)

10.Han, L., Noble, J.A.Purcher, M.: A Novel Ultrasound Indentation System for Measuring Biomechanical Propert ies of in Vivo Soft Tissue. Ultrasound in Medicine \& Biology, 29. (2003) 813-823 
10 Yi Liu1, Amy E. Kerdok1, 2, Robert D. Howe1, 2

11.Hayes, W.C., Keer, L.M., Hermann, G.Mockros, L.F.: A Mathematical Analysis for Indentation Tests of Articular Cartilage. J. Biomechanics, 5. (1972) 541-551

12.Holzapfel, G.A.: Nonlinear Solid Mechanics: A Continuum Approach for Engineering. John Wiley \& Sons Ltd., West Sussex, England (2000)

13.Hutter, R., Schmitt, K.-U.Niederer, P.: Mechanical Modeling of Soft Biological Tissues for Application in Virtual Reality Based Laparoscopy Simulators. Technology and Health Care, 8. (2000) 15-24

14.Johnson, K.L.: Contact Mechanics. Cambridge University Press, Cambridge, UK (1985)

15.Kauer, M., V. Vuskovic, J. Dual, G. SzekelyM. Bajka: Inverse Finite Element Characterization of Soft Tissues. In: Medical Image Computing and Computer-Assisted Intervention - MICCAI. (Utrecht, The Netherlands, 2001) 128-136

16. Kim, J., Tay, B., Stylopoulos, N., Rattner, D.W.Srinivasan, M.A.: Characterization of IntraAbdominal Tissues from in Vivo Animal Experiment for Surgical Simulation. In: MICCAI. 2003)

17.Krouskop, T.A., Wheeler, T.M., Kallel, F., Garra, B.S.Hall, T.: Elastic Moduli of Breast and Prostate Tissues under Compression. Ultrasonic Imaging, 20. (1998) 260-274

18. Miller, K.: Biomechanics of Soft Tissues. Med. Sci. Monit, 6. (2000) 158-167

19. Ottensmeyer, M.P.: In Vivo Data Acquisition Instrument for Solid Organ Mechanical Property Measurement. In: Medical Image Computing and Computer-Assisted Intervention MICCAI. (Utrecht, The Netherlands, 2001) 975-982

20.Plewes, D.B., Bishop, J., Samani, A.Sciarretta, J.: Visualization and Quantization of Breast Cancer Biomechanical Properties with Magnetic Resonance Elastography. Physics in Medicine and Biology, 45. (2000) 1591-1610

21.Sarvazyan, A.P., Skovoroda, A.R.Pyt'ev, Y.P.: Mechanical Introscopy - a New Modality of Medical Imaging for Detection of Breast and Prostate Cancer. In: Eighth IEEE Symposium on Computer Based Medical Systems. 1997)

22.Skovoroda, A.R., Klishko, A.N., Gusakyan, D.A., Mayevskii, Y.I., Yermilova, V.D., Oranskaya, G.A.Sarvazyan, A.P.: Quantitative Analysis of the Mechanical Characteristics of Pathologically Changed Soft Biological Tissues. Biophysics, 40. (1995) 1359-1364

23. Szekely, G., Brechbuhler, C., Dual, J.al., e.: Virtual Reality- Based Simulation of Endoscopic Surgery. Presence, 9. (2000) 310-333

24.T imoshenko, S.Goodier, J.N.: Theory of Elasticisy. McGraw-Hill, New York (1970)

25.Wellman, P.S.: Tactile Imaging. Division of Engineering and Applied Sciences. Cambridge, Harvard University (1999) 137

26.Wu, X., Downes, M.S., Goktekin, T.Tendick, F.: Adaptive Nonlinear Finite Elements for Deformable Body Simulation Using Dynamic Progressive Meshes. In: Eurographics 2001, Computer Graphics Forum, 20. 2001) 349-358

27.Yeh, W.-C., Li, P.-C., Jeng, Y.-M., Hsu, H.-C., Kuo, P.L., Li, M.L., Yang, P.M.Lee, P.H.: Elastic Modulus Measurements of Human Liver and Correlation with Pathology. Ultrasound in Medicine \& Biology, 28. (2002) 467-474

28.Zhang, M., Zheng, Y.P.Mak, A.F.: Estimating the Effective Young's Modulus of Soft Tissues from Indentation Tests - Nonlinear Finite Element Analysis of Effects of Friction and Large Deformation. Med. Eng. Phys., 19. (1997) 512-517

\section{Acknowledgements}

The authors would like to thank Parris Wellman for the use of his data, and Prof. Simona Socrate for her assistance with the modeling. 